\title{
Laproscopic intravesical vesicovaginal fistula repair after removal of an old vaginal drain tube-a rare case
}

\author{
Hafeez Rahman, Nikita Trehan, Dhruv P. Gohil*, Ajay Aggarwal
}

Sunrise Hospital, New Delhi, India

Received: 24 March 2017

Revised: 26 April 2017

Accepted: 06 May 2017

\section{*Correspondence:}

Dr. Dhruv P. Gohil,

E-mail: detartsurf@gmail.com

Copyright: () the author(s), publisher and licensee Medip Academy. This is an open-access article distributed under the terms of the Creative Commons Attribution Non-Commercial License, which permits unrestricted non-commercial use, distribution, and reproduction in any medium, provided the original work is properly cited.

\begin{abstract}
The management of vesicovaginal fistula is difficult and challenging We are presenting a rare case of a 24-year-old unmarried girl with history of vaginoplasty and multiple surgeries done in the past, with a vaginal drain tube kept for 12 years and a Vesicovaginal fistula at the bladder trigone. Patient was successfully treated with a laproscopic Intravesical vesicovaginal Fistula repair. As advances in understanding the etiology of VVF have been made, the laproscopic approach has become the gold standard. Laparoscopy allows an excellent view, good exposure of pelvic structures, provides direct access to the fistula and for repair of complex VVF that may not be amenable to vaginal repair.
\end{abstract}

Keywords: Fistula, Intravesical, Laproscopic, Repair

\section{INTRODUCTION}

Vesicovaginal fistula (VVF) is an abnormal tract extending between the bladder and the vagina allowing continuous involuntary discharge of urine into the vagina. The management of this condition is difficult and challenging. Additionally, it carries a profound psychosocial impact on the patient. It carries a high relapse and surgical failure rate. The aetiology of VVF varies and may broadly be categorized into congenital or acquired, the latter being divided into obstetric, surgical, radiation, malignant, and miscellaneous causes. The most common cause of VVF is obstructed labor. ${ }^{1}$

In the industrialized world, the most common cause $(>75 \%)$ of VVF is injury to the bladder at the time of gynecologic, urologic, or other pelvic surgery. Surgical injury to the lower urinary tract most commonly occurs in the setting of hysterectomy, whereas most of the remainders are related to general surgery procedures in the pelvis, anterior colporrhaphy or cystocele repair, anti- incontinence surgery, or other urologic procedures. ${ }^{2}$ Hereby we are presenting a rare case of a 24-year-old unmarried girl with history of vaginoplasty and multiple surgeries done in the past, with a vaginal drain tube kept for 12 years and a vesicovaginal Fistula at the bladder trigone. Patient was successfully treated with a laproscopic intravesical vesicovaginal Fistula repair.

\section{CASE REPORT}

A 24-year-old unmarried girl came to our tertiary referral centre in August 2016 with a rubber drain tube kept in vagina through which she menstrauated and complained of leakage of urine per vaginum (A VVF in place)

In November 2004, she visited a gynecologist with complaints of primary amenorrhoea and cyclical monthly pain in abdomen. She was diagnosed as a case of absent vagina with hematocolpos and absent left kidney. Her uterus, tubes and ovaries were normal. Secondary sexual characters were normal. Hematocolpos drainage with 
McIndoe's vaginoplasty (January 2005) was done. She had a rocking post-operative period with recurrent episodes of fever, pain in abdomen.

She developed a pelvic abscess (March 2005) for which she underwent a laprotomy with pelvic abscess drainage, appendicectomy, partial omentectomy, vaginal dilatation. Patient again had a hydrometrocolpos* for which drainage was done along with vaginal dilatation (July 2005). 15 days later she had leakage of urine per vaginum. A VVF was diagnosed by the treating gynaecologist.

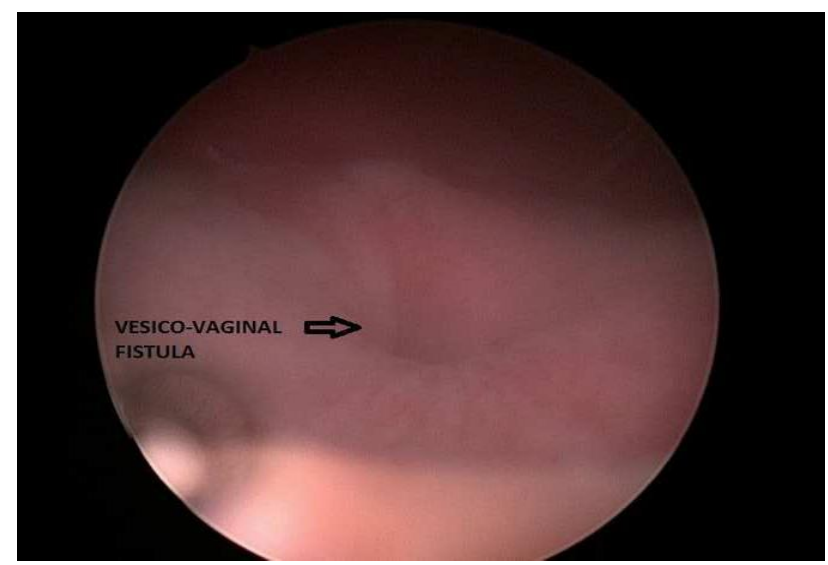

Figure 1: Cystoscopic view showing fistula site.

A vaginal drain tube was inserted under general anaaesthesia and Foley's catheter was kept for 6 weeks. Patient did not follow up with the treating gynaecologist due to personal reasons.

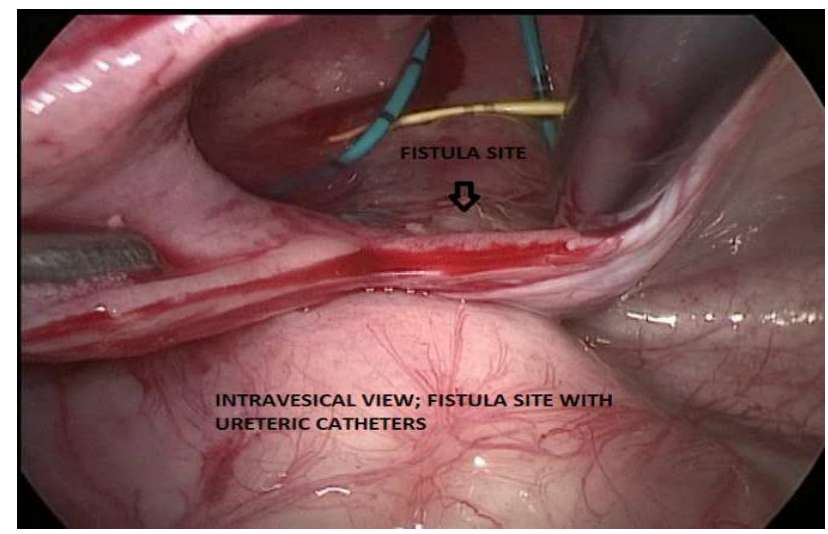

Figure 2: Intravesical view 1.

Patient used to menstruate normally through vaginal drain tube and had minimal leakage of urine per vaginum (probably the fistula was blocked physically by the vaginal drain tube for 10 years). When she came to us in August 2016 her intraoperative findings were as followsDrain tube kept in vagina was removed and sent for culture and sensitivity. Hysteroscopy-small cervical polyp was present, uterine cavity was normal, bilateral ostia visualized, polypectomy was done.

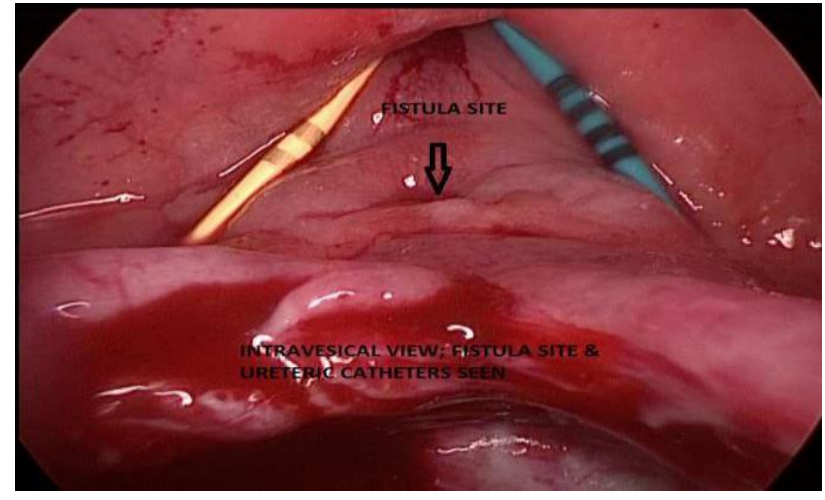

Figure 3: Intravesical view 2.

A vesicovaginal fistula was present at the bladder trigone which was confirmed on cystoscopy. On laproscopyuterus was of normal size, tubes and ovaries were normal. Adhesions were present over abdominal wall bilaterally. adhesiolysis was done.

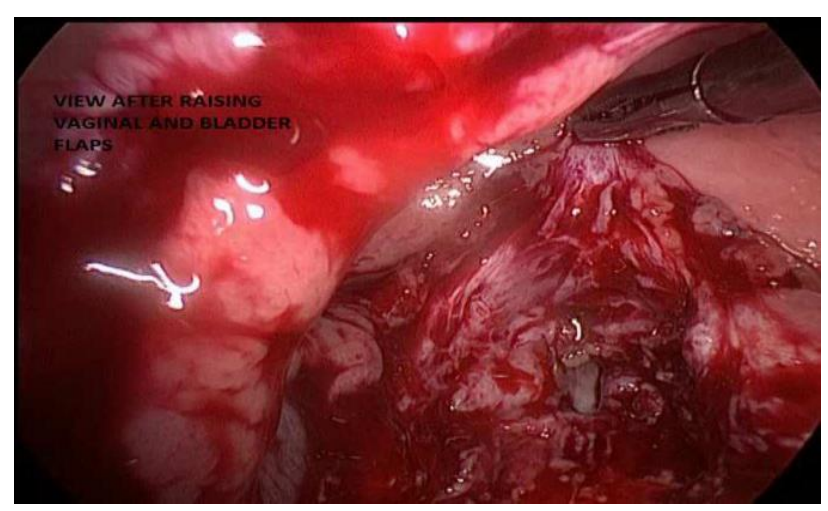

Figure 4: View after raising flaps.

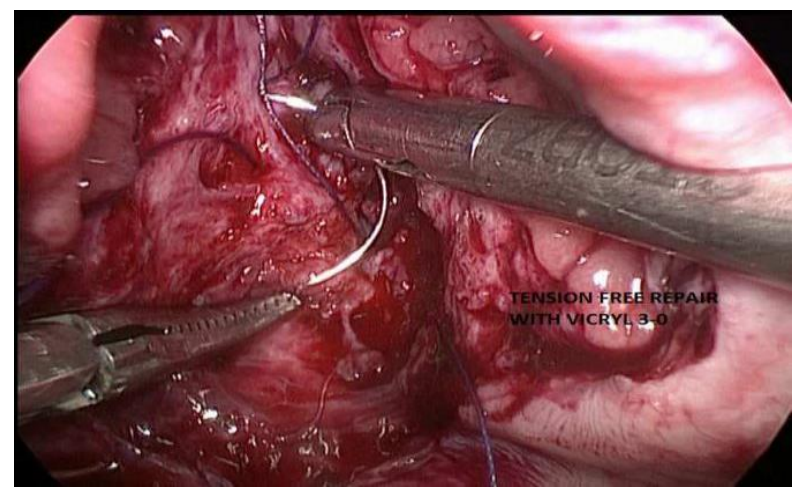

Figure 5: Tension free repair.

Intravesical repair of VVF was done. After the bladder was opened, a VVF of $1 \mathrm{~cm}$ size was seen at the trigonal area with indurated edges. Bilateral ureteric stenting was done. Vaginal flap and bladder flap was raised and the two were separated. Edges were freshened. Laproscopic closure of vaginal defect was done with vicryl 4-0. Evicel (Johnson and Johnson, Human Fibrin sealant) was inserted through vagina. Bladder defect was then closed over it with vicryl 4-0. 
Hemostasis was checked. Patient was started on broad spectrum antibiotics. Bladder was catheterized for 6 weeks. Patient recovered well post operatively, her urinary complaints resolved and got her menses normally.

\section{DISCUSSION}

Principles of surgical repair of VVF include optimal tissue condition (in terms of vascular supply, infection, inflammation), option of complete excision of fistulous tract, a tension-free, water-tight, multilayered closure with avoidance of overlapping suture lines, interposition of healthy vascularised tissue (like omentum) between the bladder and vaginal suture, continuous postoperative bladder drainage for 6 weeks and broad spectrum antibiotics.

This was an extremely rare case of a VVF in a young girl with history of vaginoplasty done along with multiple surgeries and a vaginal drain tube kept in situ. Lee et al recommended an abdominal approach for certain indications, namely 1) inadequate exposure related to a high or retracted fistula in a narrow vagina, 2) close proximity of the fistulous tract to the ureter, 3) associated pelvic pathology and 4) multiple fistulas. ${ }^{3}$ The approach of management of Vesicovaginal Fistula (VVF) has been shifted from a trans abdominal/vaginal approach to a minimally invasive (laproscopic) approach. According to the study of Chau-Su Ou et al in 2004 on comparison of results between a series of laparoscopic repairs, transabdominal open repairs, transvaginal repairs, laparoscopic VVF repair was feasible and may result in lower morbidity, shorter hospital stay, and quicker recovery than the abdominal or transvaginal approaches. ${ }^{4}$

However, when the fistula is high lying on the posterior bladder wall and the vagina is severely scarred, vaginal exposure may be difficult. ${ }^{5}$ The intravesical approach may provide a clear operative field to close the opening of the fistulous tract in the bladder without contacting the perivesical infected and inflamed tissue e.g. cases of trigonal fistulas close to ureteric orifices, when adequate flaps cannot be raised, when there is perivesical scarring as in cases of previous surgeries.

Also, in present case which was a previously created McIndoes Vaginoplasty, the vaginal space and tissue flaps were limited and hence a decision for intravesical repair was taken. Interpositions like vasuclarised omental grafts, Martius grafts and fibrin glue are used for better results as relapse and failures are common. The interposition of flaps is considered to be a protective factor for the recurrent cases. ${ }^{6}$ In our case, we used fibrin glue Evicel (Johnson and Johnson, an adjunct to hemostasis for use in patients undergoing surgery, does not contain aprotinin or bovine derivatives and does not expose patients to the risks associated with aprotinin). Fibrin glue promotes healing through its effects on fibroblasts and collagen synthesis. It is a feasible option as it prevents fibrosis. It is biodegradable and is absorbed within weeks of its application.

\section{CONCLUSION}

As advances in understanding the etiology of VVF have been made, the laproscopic approach has become the gold standard. Laparoscopy allows an excellent view, good exposure of pelvic structures, provides direct access to the fistula and for repair of complex VVF that may not be amenable to vaginal repair. In this case, as the fistula was in the trigonal region with previous history of vaginoplasty and multiple surgeries (inadequate vaginal flap tissue available), we proceeded with an intravesical approach. The above-mentioned method with fibrin glue interposition is a reproducible method and yields better results. Hydrometrocolpos is characterized by an expanded fluid filled vaginal cavity with associated distention of uterine cavity.

Funding: No funding sources

Conflict of interest: None declared

Ethical approval: Not required

\section{REFERENCES}

1. Tahzib F. Epidemiological determinants of vesicovaginal fistulas. $\mathrm{Br} \quad \mathrm{J}$ Obstet Gynaecol. 1983;90:387-91.

2. Armenakas NA, Pareek G, Fracchia JA. Iatrogenic bladder perforations: long-term follow-up of 65 patients. J Am Coll Surg. 2004;198:78-82.

3. Lee RA, Symmonds RE, Williams TJ. Current status of genitourinary fistula. Obstet Gynecol. 1998;72: 313.

4. Ou CS, Huang UC, Tsuang M, Rowbotham R. J Laparoendosco Advanc Surg Tech. 2004;14(1):17-21.

5. Shlomo R. Editorial comment: early versus late repair of vesicovaginal fistulas: vaginal and abdominal approaches. J Urol. 1995; 153:1112.

6. Ockrim JL, Greenwell TJ, Foley CL, Wood DN, Shah PJR. A tertiary experience of vesico-vaginal and urethro-vaginal fistula repair: factors predicting success. BJU Int. 2009; 103:1122-6.

Cite this article as: Rahman $\mathrm{H}$, Trehan N, Gohil DP, Aggarwal A. Laproscopic intravesical vesicovaginal fistula repair after removal of an old vaginal drain tube-a rare case. Int J Reprod Contracept Obstet Gynecol 2017;6:2654-6. 